\title{
Impacts of Outdoor Air Pollution on Human Semen Quality: A Meta-Analysis and Systematic Review
}

\author{
Jianzhong Zhang, Zhonglin Cai, Chengquan Ma, Jian Xiong, and Hongjun Li iD \\ Department of Urology, Peking Union Medical College Hospital, Peking Union Medical College, Chinese Academy of \\ Medical Sciences, Beijing 100730, China \\ Correspondence should be addressed to Hongjun Li; lihongjun@pumch.cn
}

Received 19 March 2020; Accepted 16 April 2020; Published 28 April 2020

Academic Editor: Heide Schatten

Copyright (c) 2020 Jianzhong Zhang et al. This is an open access article distributed under the Creative Commons Attribution License, which permits unrestricted use, distribution, and reproduction in any medium, provided the original work is properly cited.

\begin{abstract}
Introduction. Several studies have explored the association between outdoor air pollution and semen quality. However, the results were inconsistent. We performed the current meta-analysis to evaluate the role of outdoor air pollution in semen quality. Material and Methods. Databases including PubMed, Web of Science, and Embase were searched to identify relevant studies. Relative data in participants under higher exposure and lower exposure to air pollution were extracted. Pooled weighted mean differences (WMDs) with corresponding 95\% confidence intervals (CIs) were utilized to assess the effects of outdoor air pollution on semen quality. In addition, trial sequential analyses (TSAs) were performed to obtain a more comprehensive assessment of analyses. Results. A total of 11 studies with 4562 males were enrolled in the current meta-analysis. Higher air pollution levels were associated with significant decreases in semen volume (WMD: $-0.16,95 \%$ CI: -0.27 to -0.05), sperm concentration (WMD: $-5.52,95 \%$ CI: -9.88 to -1.16), progressive motility (WMD: $-6.23,95 \%$ CI: -11.64 to -0.81 ), total motility (WMD: $-7.65,95 \%$ CI: -14.09 to -1.20 ), and normal sperm morphology rate (WMD: $-3.71,95 \%$ CI: -5.59 to -1.82). In addition, the DNA fragmentation index significantly increased (WMD: 4.11, 95\% CI: 1.94 to 6.29). Conclusions. Air pollution is associated with decreased semen volume, sperm concentration, motility, and normal morphology rate.
\end{abstract}

\section{Introduction}

According to the definition of the International Organization for Standardization (ISO), air pollution usually refers to the phenomenon that harmful or excessive quantities of substances enter the atmosphere due to human activities or natural processes. When the pollutants accumulate to enough concentration and sustained for enough time, air pollution will significantly impair the health of human beings. It can result in various diseases including cardiovascular and lung diseases, neurologic disorders, and infertility [1-5]. Recently, various studies have explored the effects of air pollution on male fertility $[6,7]$.

Human semen quality has been degraded for decades. Several studies have demonstrated that exposure to toxicants or air pollutants, electromagnetic waves from cell phones, obesity, drinking, smoking, psychological stress hypertension, and diabetes can be potential causes of this degradation $[6,8-14]$. Considering the large number of affected populations, outdoor air pollution has become the hotspot recently. However, the specific role of air pollution in semen quality remains unclear. Epidemiologic studies have demonstrated nonsignificant or contrary results. Several studies demonstrated that air pollution can significantly reduce the sperm concentration [15-18] and total sperm count [16-18], but several studies did not show significant results. Concerning the sperm motility, air pollution was reported associated with decreased progressive sperm motility $[15,16,19-21]$ and total sperm motility [16, 19-22]. However, some other studies did not demonstrate significant results.

Based on the data in the previous published studies, the current meta-analysis was performed to explore the overall impacts of air pollution on semen quality. 


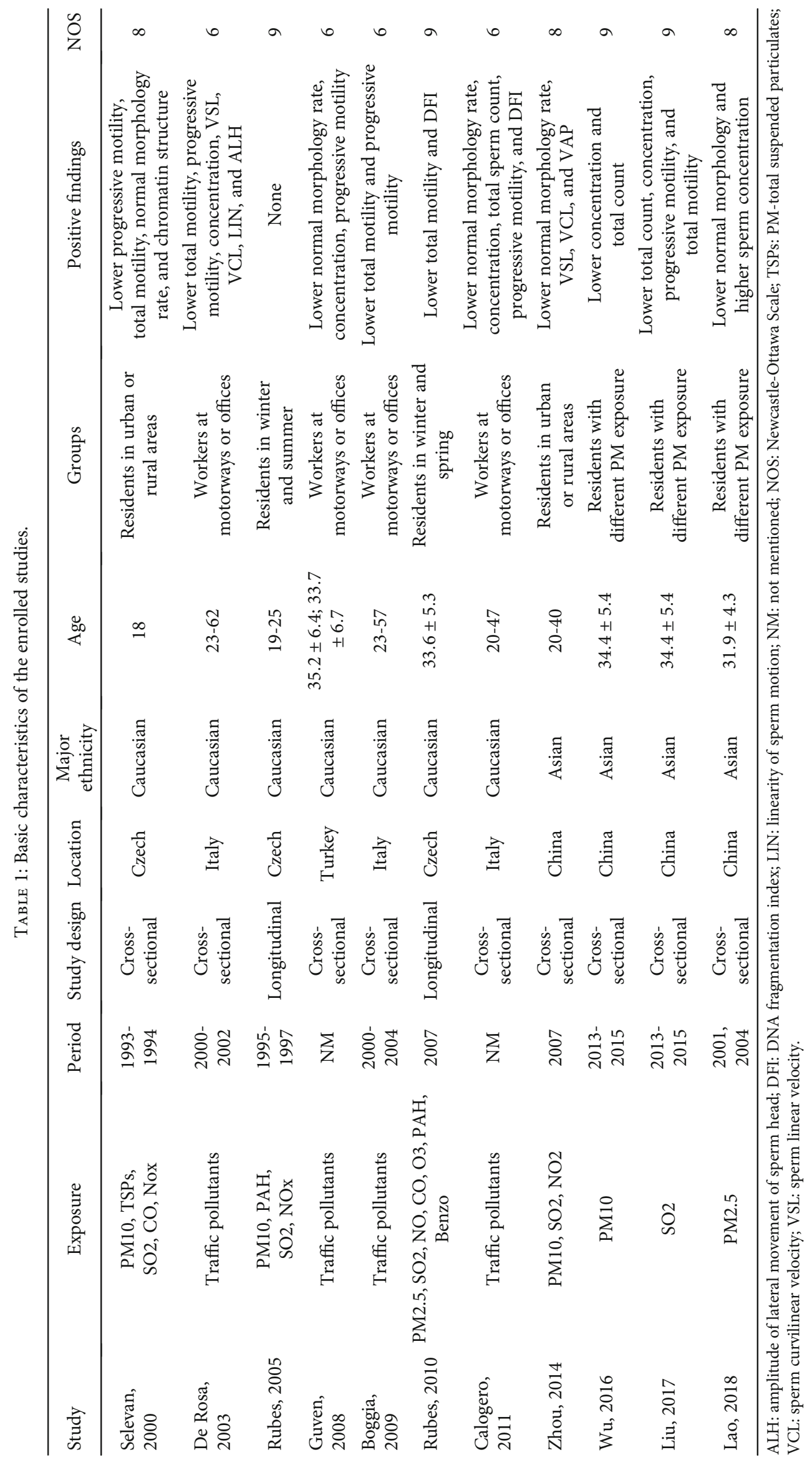




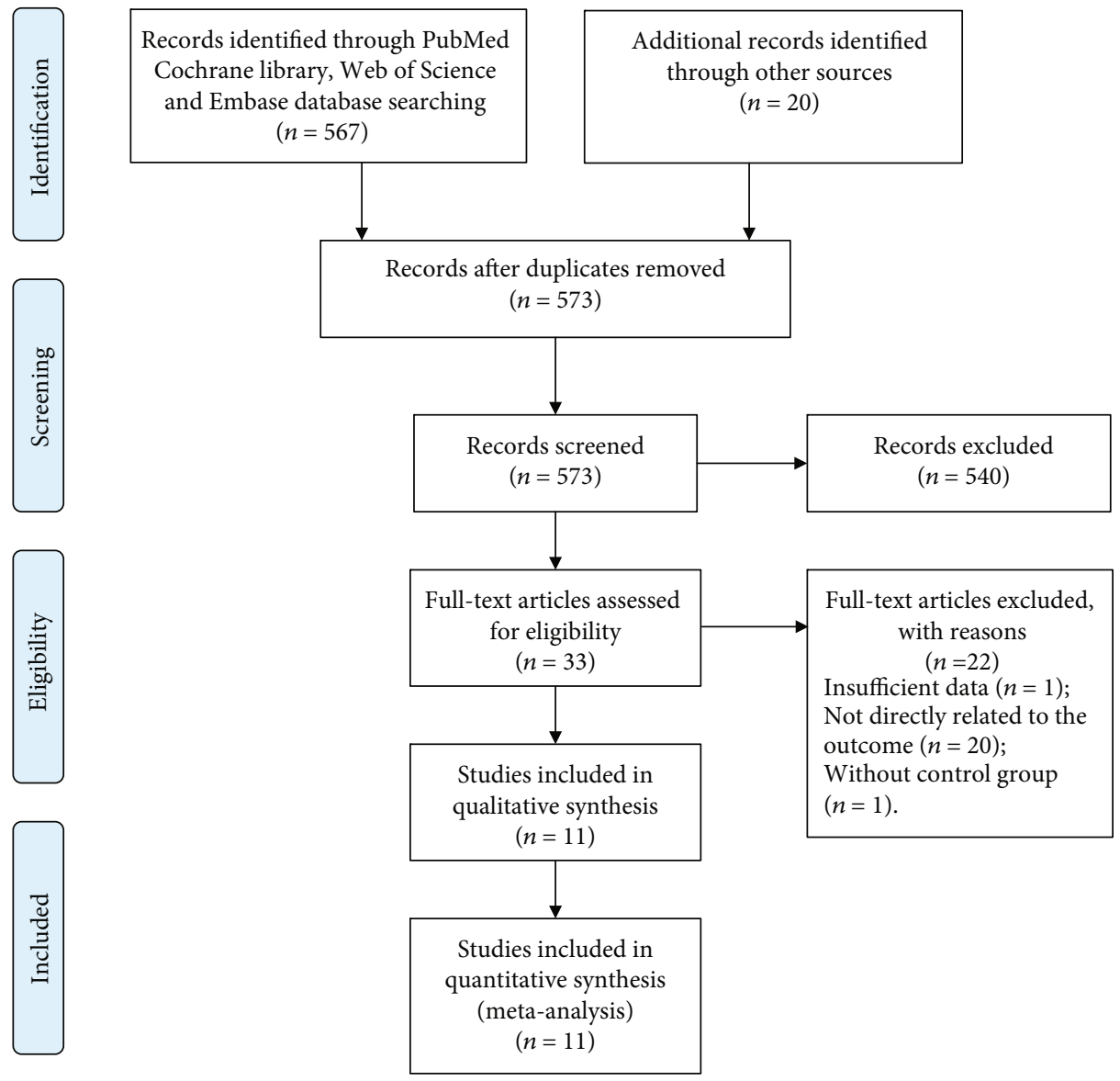

FIGURE 1: Flow diagram of the study selection process.

\section{Materials and Methods}

This study was strictly reported based on the PRISMA (Preferred Reporting Items for Systematic Review and Meta-analyses) statement [23]. The protocol of the present study was described previously [24] and registered in the international prospective register of systematic reviews (registration number CRD42019126060). We used the same research methods in the current study.

The quality of the enrolled studies was evaluated by Newcastle-Ottawa Scale (NOS) star system (range, 0 to 9 stars), which focuses on three broad perspectives: the selection of the study groups, the comparability of the groups, and the ascertainment of either the exposure or outcome of interest. The number of stars is positively associated with the quality of the study. Overall, the enrolled studies rated from 6 to 9 stars (Table 1).

\section{Results}

3.1. Basic Characteristics of the Enrolled Studies. The study selection process was shown in Figure 1. In total, eleven studies with 4652 males met the inclusion criteria and were enrolled in the current meta-analysis [15-22, 25-27]. Notably, the outdoor air pollutants varied between the included studies. Four studies explored the role of traffic pollutants in male fertility and did not analyze the composition of the air pollutants. Among the 11 enrolled studies, nine were cross-sectional studies while the other two were longitudinal studies. Seven articles mainly focused on Caucasians and four focused on Asians. Participants were divided into different groups based on the extent of exposure to air pollution. Five, two, and four studies were grouped together according to the location, climate, and working conditions of the participants, respectively. Details of the aforementioned data are listed in Table 1.

3.2. The Effects of Outdoor Air Pollution on Sperm Parameters. All eleven studies reported the role of outdoor air pollution in sperm concentration. Among them, six [15, $19,20,22,25,26]$ and five $[16-19,25]$ studies further explored the alterations in semen volume and total sperm count, respectively. The results indicated that higher air pollution levels were associated with significant decreases in semen volume (WMD: $-0.16,95 \% \mathrm{CI}:-0.27$ to -0.05 ) (Figure 2(a)) and sperm concentration (WMD: $-5.52,95 \%$ CI: -9.88 to -1.16 ) (Figure 2(b)). Notably, the decrease in total sperm count, which was obtained by multiplying semen volume by sperm concentration, was not significant (WMD: $-38.19,95 \%$ CI: -82.89 to 6.50 ) (Figure $2(\mathrm{c})$ ). This may have partly resulting from the limited sample size.

Six studies explored the association between air pollution and normal sperm morphology rate [16, 19, 22, 25-27]. The pooled results demonstrated a significant decrease in normal 


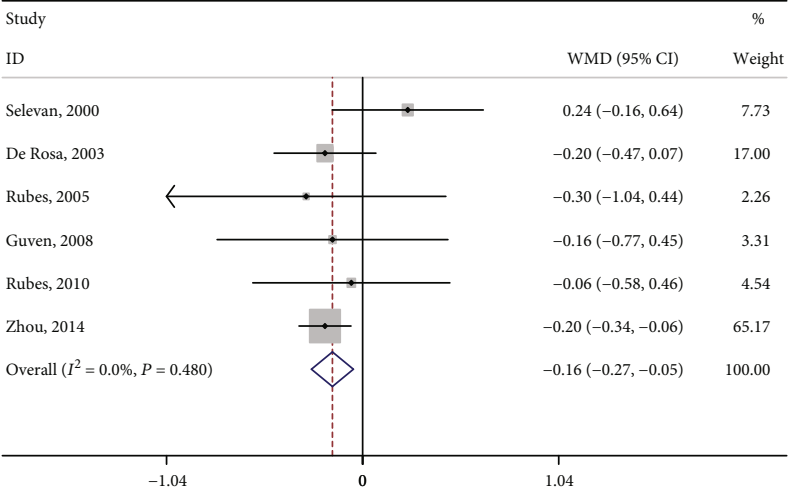

(a)

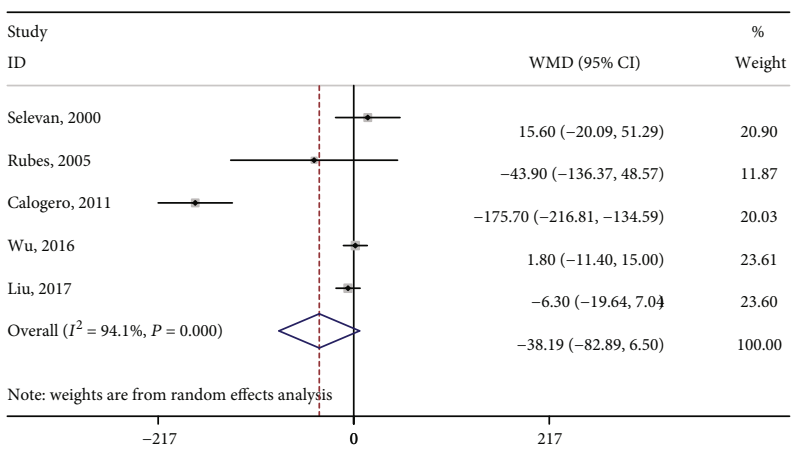

(c)

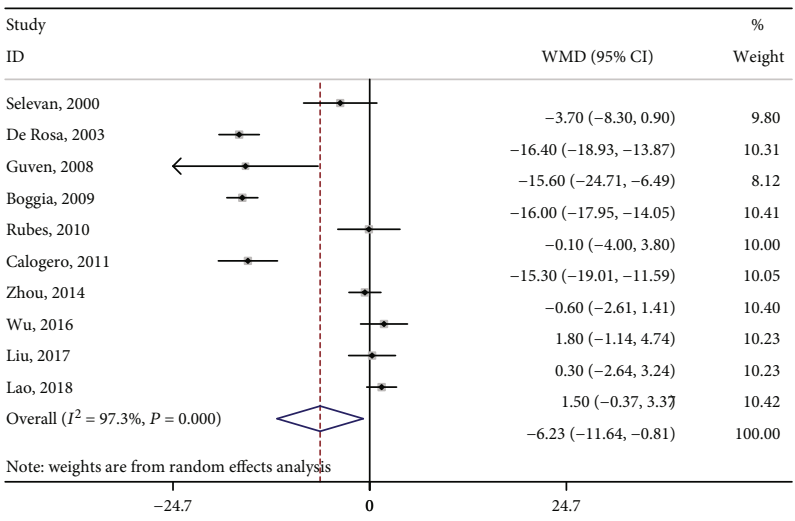

(e)

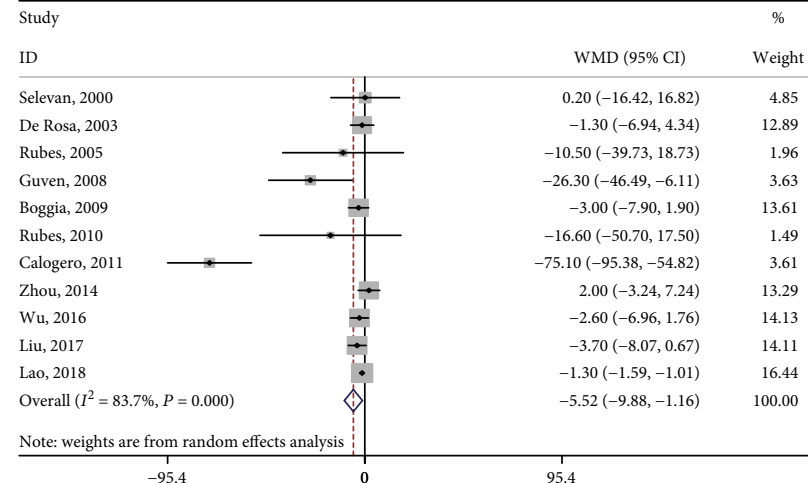

(b)

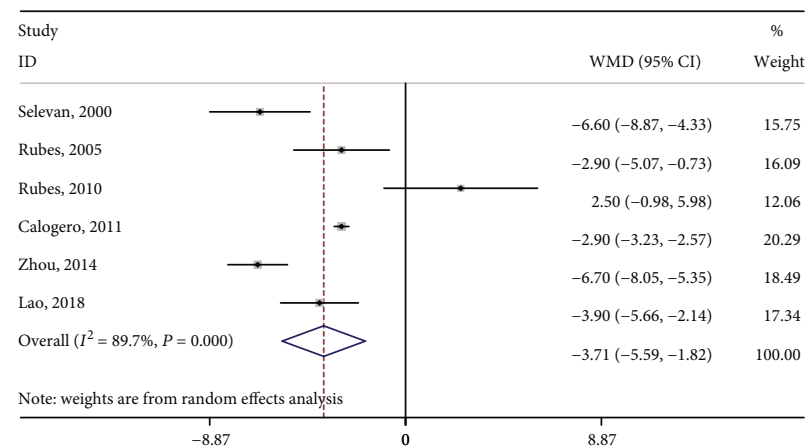

(d)

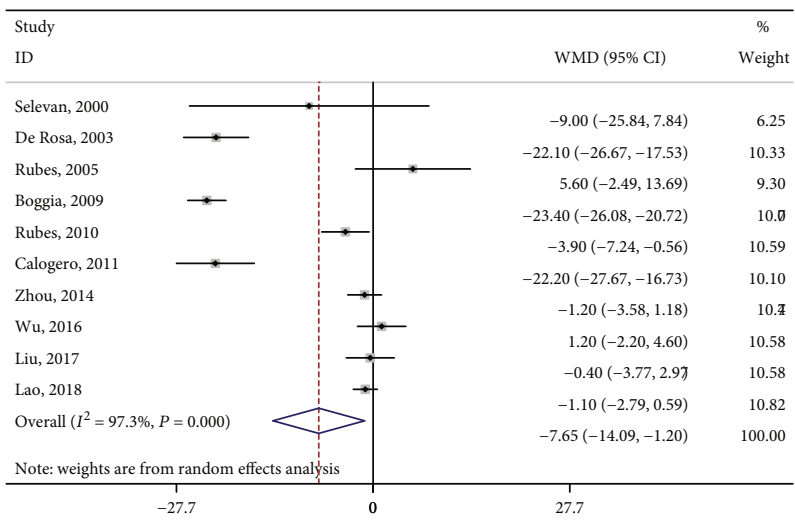

(f)

FigURE 2: Forest plots of merged analyses of effects on sperm parameters by outdoor air pollution. (a-e) Forests plots of merged analyses of semen volume, sperm concentration, total sperm count, normal morphology rate, progressive motility, and total sperm motility, respectively.

morphology (WMD: $-3.71,95 \%$ CI: -5.59 to -1.82 ) (Figure 2(d)). Ten studies explored the association between outdoor air pollution and sperm motility [15-22, 26, 27]. The results indicated that air pollution was associated with significant decreases in progressive motility (WMD: -6.23, 95\% CI: -11.64 to -0.81 ) (Figure 2(e)) and total motility (WMD: -7.65 , 95\% CI: -14.09 to -1.20 ) (Figure 2(f)). In addition, the DNA fragmentation index significantly increased based on the pooled result from four studies [16, 19, 22, 25] (WMD: 4.11, 95\% CI: 1.94 to 6.29) (Figure 3(a)). Details of the aforementioned information of each enrolled study are listed in Table 2.

CASA measures were provided in four studies [19, 20, $25,26]$, and our meta-analysis demonstrated nonsignifi- cant decreases in VCL (WMD: $-1.59,95 \%$ CI: -14.71 to 11.53) (Figure 3(b)), VSL (WMD: -3.35 , 95\% CI: -11.16 to 4.46 ) (Figure $3(\mathrm{c})$ ), and LIN (WMD: $-11.51,95 \% \mathrm{CI}$ : -25.38 to 2.36) (Figure 3(d)). Detailed information concerning CASA measures of each enrolled study is listed in Table 3.

3.3. Trial Sequential Analysis Results. The TSA results indicated sufficient evidence that outdoor air pollution reduced semen volume (Figure 4(a)), sperm concentration (Figure 4(b)), normal morphology rate (Figure 4(c)), and total motility (Figure 4(d)). However, analysis of progressive motility showed a negative result, indicating that inaccuracy might exist (data not shown). Further studies are required 


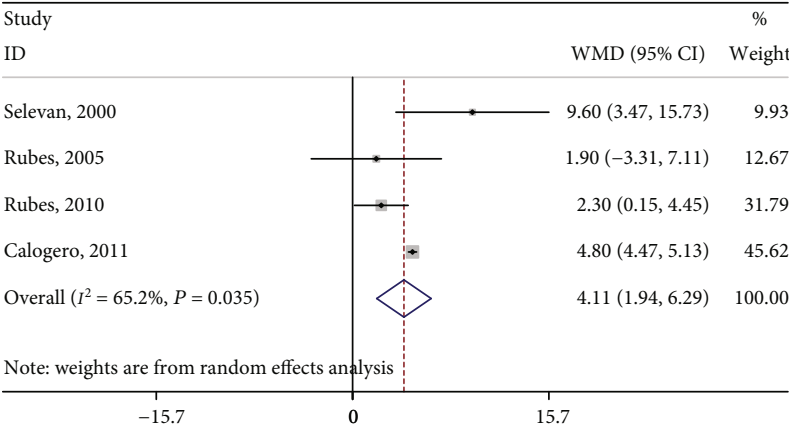

(a)

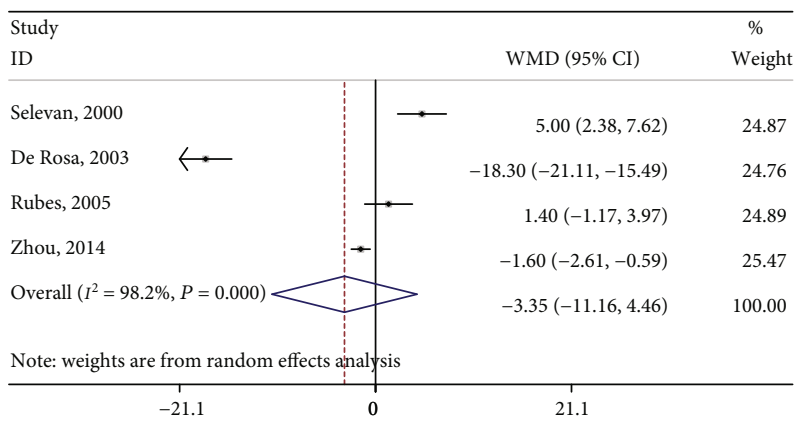

(c)

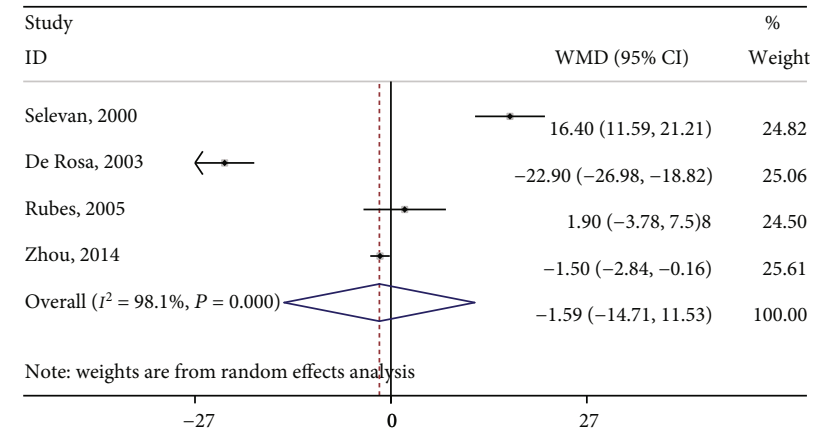

(b)

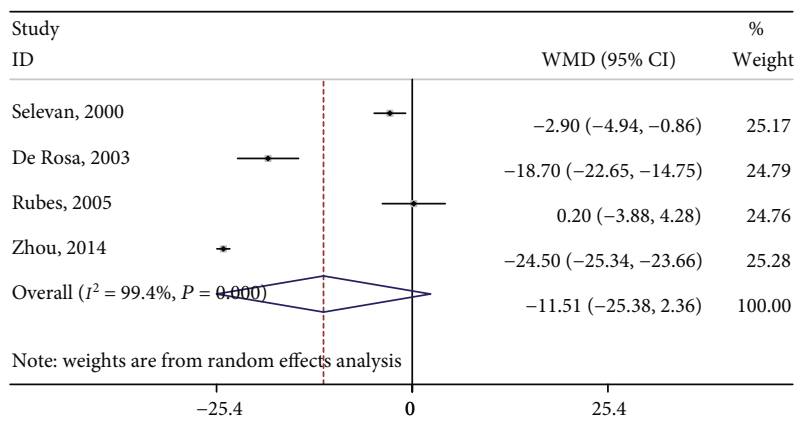

(d)

FIgURe 3: Forest plots of merged analyses of effects on DFI and CASA measures by outdoor air pollution. (a-d) Forests plots of merged analyses of DFI, VCL, VSL, and LIN, respectively.

to explore the role of outdoor air pollution in sperm progressive motility.

3.4. Sensitivity Analysis. The influence of individual studies on the pooled WMDs was evaluated by sensitivity analyses (Figure S1). No significant alterations in pooled WMDs were observed after any single study was omitted, demonstrating that the results were robust.

3.5. Publication Bias. The results of Egger's linear regression tests demonstrated no potential publication bias of the enrolled studies (Semen volume: $P=0.433$; sperm concentration: $P=0.124$; total sperm count: $P=0.372$; progressive motility: $P=0.854$; total motility: $P=0.495$; normal morphology rate: $P=0.528$; DFI: $P=0.689$; VCL: $P=0.984$; VSL: $P=0.795$; and LIN: $P=0.260$ ). In addition, evidence of obvious asymmetry was not found in the funnel plots (Figure S2).

\section{Discussion}

Testicular function and sperm development can be affected by exposure to various environmental pollutants, including isoflavones, heavy metals, chlorination disinfection byproducts in water, organic solvents, and particulate air pollution [14]. Recently, various studies focused on other harmful environment urban factors, especially the electromagnetic waves from cell phones and stations, can also decrease semen quality and their negative influence cannot be objectively separated from the other environmental pollutants $[12,13]$.
The effect of pollutants on sperm quality could be evaluated in humans or in laboratory animals. Several animal studies have been performed to investigate the negative effects of air pollution on semen parameters. Prenatal exposure to diesel exhaust has been associated with a significant reduction in daily sperm production, multinucleated giant cells in the seminiferous tubules, partial vacuolation of the seminiferous tubules, and elevated follicle-stimulating hormone receptor (FSHR) mRNA expression in mice [28]. The biological mechanisms of the effects of air pollution on semen quality remain uncertain, and relevant research is limited. One possible mechanism is disorder in the hypothalamic pituitary axis. Particulate matter, i.e., microscopic solid or liquid matter suspended in the atmosphere of Earth, can carry multiple trace elements and polycyclic aromatic hydrocarbons (PAHs). PAHs are a group of compounds that include several endocrine disruptors and can influence sexual hormones by interfering with the hypothalamic pituitary axis [29]. In addition, PAHs can directly impair spermatogenesis [30]. Several studies have demonstrated that the reactive metabolites of $\mathrm{PM}_{10}$ and $\mathrm{PM}_{2.5}$ can reach the testes and cause increased mitochondrial dysfunction, DNA fragmentation, and cell apoptosis $[30,31] . \mathrm{O}_{3}$-induced oxidative stress is another possible mechanism. Sperm exist in a balanced physiological environment of reactive oxygen species (ROS) and antioxidants. $\mathrm{O}_{3}$ may result in inflammation in the male genital tract and formation of circulating toxic species and ROS. Excessive amounts of ROS can subsequently impair the integrity of the DNA in the sperm nucleus and accelerate the process of sperm apoptosis $[32,33]$. Luo et al. 
TABLe 2: Primary outcomes of the enrolled studies.

\begin{tabular}{|c|c|c|c|c|c|c|c|c|}
\hline \multirow[t]{2}{*}{ Study } & \multicolumn{2}{|c|}{ Sample size } & \multicolumn{2}{|c|}{ Semen volume $(\mathrm{mL})$} & \multicolumn{2}{|c|}{$\begin{array}{c}\text { Sperm concentration } \\
\left(10^{6} / \mathrm{mL}\right)\end{array}$} & \multicolumn{2}{|c|}{ Total count $\left(10^{6}\right)$} \\
\hline & High & Low & High & Low & High & Low & High & Low \\
\hline Selevan, 2000 & 47 & 162 & $2.2 \pm 1.3$ & $2.0 \pm 1.1$ & $60.1 \pm 46.7$ & $59.9 \pm 64.3$ & $129.1 \pm 103.1$ & $113.5 \pm 130.7$ \\
\hline De Rosa, 2003 & 85 & 85 & $2.5 \pm 0.9$ & $2.7 \pm 0.9$ & $32.4 \pm 22.1$ & $33.7 \pm 14.7$ & NM & $\mathrm{NM}$ \\
\hline Rubes, 2005 & 36 & 36 & $3.0 \pm 1.7$ & $3.3 \pm 1.5$ & $81.6 \pm 42.09$ & $92.1 \pm 79.0$ & $234.2 \pm 141.1$ & $278.1 \pm 245.4$ \\
\hline Guven, 2008 & 38 & 35 & $3.2 \pm 1.3$ & $3.4 \pm 1.4$ & $44.6 \pm 36.3$ & $70.9 \pm 50.0$ & $\mathrm{NM}$ & NM \\
\hline Boggia, 2009 & 100 & 64 & $\mathrm{NM}$ & NM & $34.3 \pm 20.3$ & $37.3 \pm 11.7$ & $\mathrm{NM}$ & $\mathrm{NM}$ \\
\hline Rubes, 2010 & 47 & 47 & $3.2 \pm 1.3$ & $3.2 \pm 1.3$ & $134.2 \pm 84.1$ & $150.8 \pm 84.6$ & NM & $\mathrm{NM}$ \\
\hline Calogero, 2011 & 36 & 32 & NM & NM & $24.1 \pm 15.4$ & $99.2 \pm 56.7$ & $64.9 \pm 43.3$ & $240.6 \pm 111.4$ \\
\hline Zhou, 2014 & 429 & 917 & $2.3 \pm 1.1$ & $2.5 \pm 1.4$ & $79.4 \pm 46.2$ & $77.4 \pm 44.6$ & NM & $\mathrm{NM}$ \\
\hline Wu, 2016 & 367 & 349 & $\mathrm{NM}$ & NM & $39.4 \pm 29.1$ & $42.0 \pm 30.3$ & $104.4 \pm 82.9$ & $102.6 \pm 96.4$ \\
\hline Liu, 2017 & 370 & 327 & $\mathrm{NM}$ & NM & $39.4 \pm 27.3$ & $43.1 \pm 31.1$ & $108.4 \pm 82.0$ & $114.7 \pm 96.0$ \\
\hline Lao, 2018 & 535 & 501 & NM & $\mathrm{NM}$ & $40.6 \pm 2.5$ & $41.9 \pm 2.3$ & $\mathrm{NM}$ & $\mathrm{NM}$ \\
\hline \multirow[t]{2}{*}{ Study } & \multicolumn{2}{|c|}{$\begin{array}{c}\text { Progressive motility } \\
\text { (PR, \%) }\end{array}$} & \multicolumn{2}{|c|}{$\begin{array}{l}\text { Total motility } \\
(\mathrm{PR}+\mathrm{NP}, \%)\end{array}$} & \multicolumn{2}{|c|}{ Normal morphology (\%) } & \multicolumn{2}{|c|}{ SCSA-DFI (\%) } \\
\hline & High & Low & High & Low & High & Low & High & Low \\
\hline Selevan, 2000 & $32.5 \pm 13.2$ & $36.2 \pm 17.1$ & $41.6 \pm 40.4$ & $50.6 \pm 79.6$ & $13.2 \pm 6.5$ & $19.8 \pm 8.5$ & $28.8 \pm 20.4$ & $19.2 \pm 12.2$ \\
\hline De Rosa, 2003 & $12.3 \pm 11.0$ & $28.7 \pm 4.6$ & $34.7 \pm 20.2$ & $56.8 \pm 7.4$ & NM & NM & $\mathrm{NM}$ & $\mathrm{NM}$ \\
\hline Rubes, 2005 & NM & NM & $68.3 \pm 12.1$ & $62.7 \pm 21.6$ & $8.4 \pm 2.6$ & $11.3 \pm 6.1$ & $15.4 \pm 12.6$ & $13.5 \pm 9.8$ \\
\hline Guven, 2008 & $54.7 \pm 23.6$ & $70.3 \pm 15.6$ & $\mathrm{NM}$ & NM & NM & NM & NM & $\mathrm{NM}$ \\
\hline Boggia, 2009 & $15.0 \pm 7.4$ & $31.0 \pm 5.3$ & $37.0 \pm 11.2$ & $60.4 \pm 6.3$ & NM & NM & NM & NM \\
\hline Rubes, 2010 & $58.0 \pm 9.9$ & $58.1 \pm 9.4$ & $70.5 \pm 8.2$ & $74.4 \pm 8.3$ & $21.3 \pm 9.8$ & $18.8 \pm 7.2$ & $12.4 \pm 5.8$ & $10.1 \pm 4.8$ \\
\hline Calogero, 2011 & $12.4 \pm 8.7$ & $27.7 \pm 6.9$ & $29.6 \pm 12.8$ & $51.8 \pm 10.2$ & $17.2 \pm 0.8$ & $20.1 \pm 0.6$ & $9.3 \pm 0.9$ & $4.5 \pm 0.4$ \\
\hline Zhou, 2014 & $51.7 \pm 17.5$ & $52.3 \pm 17.5$ & $69.8 \pm 20.9$ & $71.0 \pm 20.4$ & $23.5 \pm 11.5$ & $30.2 \pm 12.3$ & NM & $\mathrm{NM}$ \\
\hline Wu, 2016 & $38.8 \pm 17.7$ & $37.0 \pm 22.1$ & $45.8 \pm 20.6$ & $44.6 \pm 25.4$ & NM & NM & $\mathrm{NM}$ & $\mathrm{NM}$ \\
\hline Liu, 2017 & $38.9 \pm 19.6$ & $38.6 \pm 19.9$ & $45.9 \pm 22.5$ & $46.3 \pm 22.8$ & NM & NM & NM & NM \\
\hline Lao, 2018 & $48.4 \pm 15.4$ & $46.9 \pm 15.3$ & $65.3 \pm 14.0$ & $66.4 \pm 13.7$ & $67.9 \pm 15.2$ & $71.8 \pm 13.7$ & NM & NM \\
\hline
\end{tabular}

NM: not mentioned.

TABLE 3: CASA measures of the enrolled studies.

\begin{tabular}{lcccccc}
\hline Study & VCL $(\mu \mathrm{m} / \mathrm{s})$ & & VSL $(\mu \mathrm{m} / \mathrm{s})$ & \multicolumn{2}{c}{ LIN $(\%)$} \\
\hline Selevan, 2000 & High & Low & High & Low & High & Low \\
De Rosa, 2003 & $107.8 \pm 12.1$ & $91.4 \pm 21.7$ & $48.3 \pm 7.4$ & $43.3 \pm 10.0$ & $44.7 \pm 5.6$ & $47.6 \pm 8.2$ \\
Rubes, 2005 & $29.7 \pm 18.4$ & $52.6 \pm 5.5$ & $16.1 \pm 12.0$ & $34.4 \pm 5.5$ & $47.1 \pm 15.6$ & $65.8 \pm 10.1$ \\
Zhou, 2014 & $72.8 \pm 11.3$ & $70.9 \pm 13.2$ & $36.4 \pm 4.7$ & $35.0 \pm 6.3$ & $52.4 \pm 8.1$ & $52.2 \pm 9.5$ \\
\hline
\end{tabular}

LIN: linearity of sperm motion; VCL: sperm curvilinear velocity; VSL: sperm linear velocity.

demonstrated that gasoline exhaust can cause significant reduction in $\alpha 6$-integrin and $\beta 1$-integrin in the rat testes, which may be a cause of decreased semen quality [34].

A large number of epidemiologic studies have explored the associations between outdoor air pollution and semen quality. However, the results were inconsistent. The current meta-analysis was performed to obtain conclusive results by pooling all qualified data. The results indicated that outdoor air pollution can significantly impair semen quality by increasing sperm DFI and decreasing semen volume, sperm concentration, motility, and normal morphology rate.

Notably, although semen volume and sperm concentration significantly decreased in participants with higher exposure to air pollution was revealed, the decrease in total sperm count, which is obtained by multiplying semen volume by sperm concentration, was not significant. There were several 


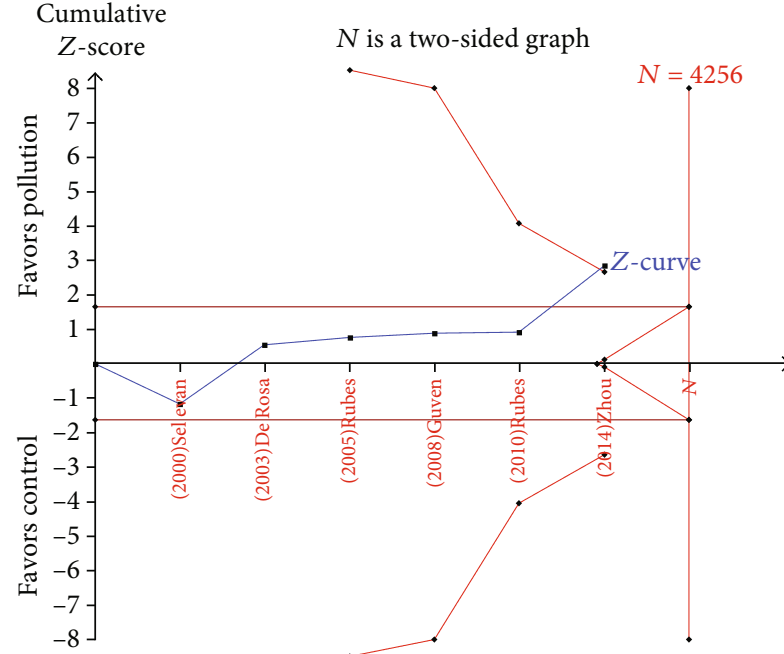

(a)

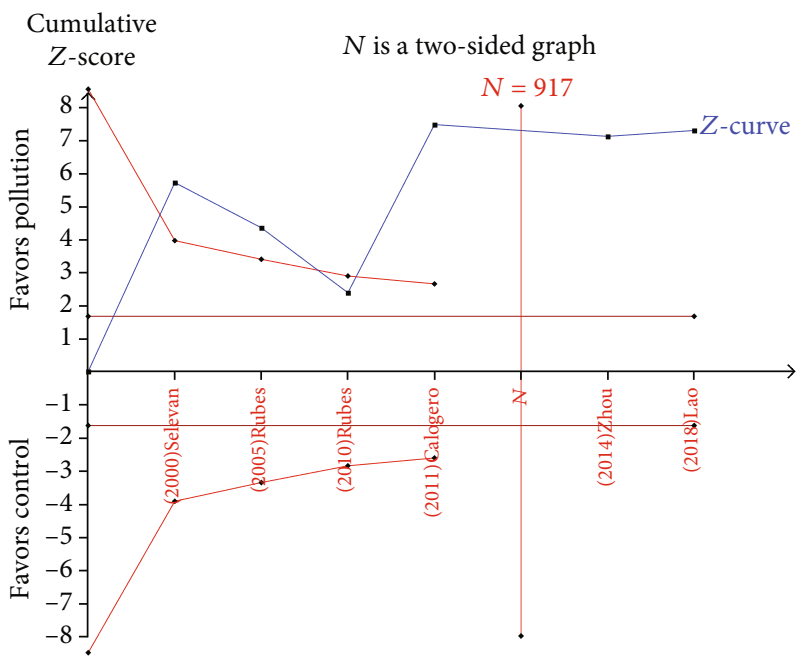

(c)

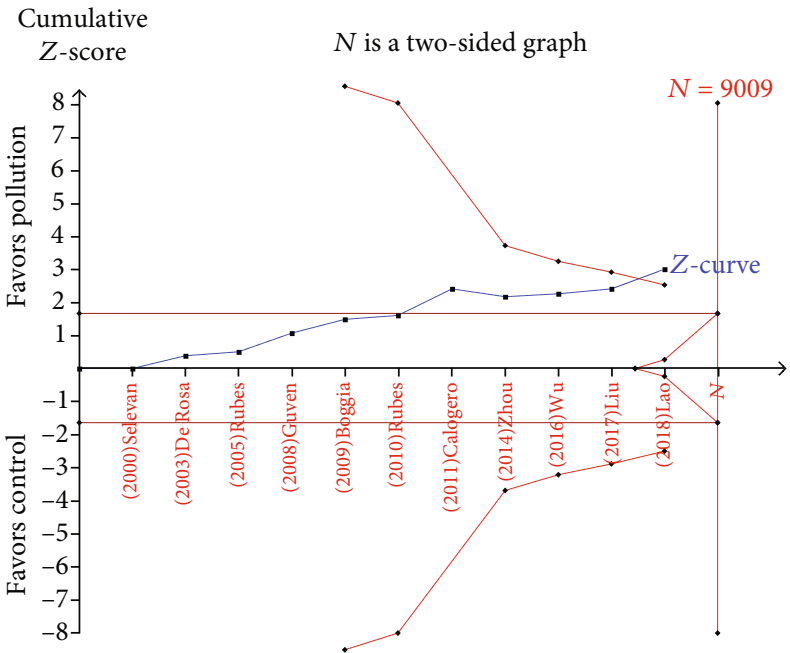

(b)

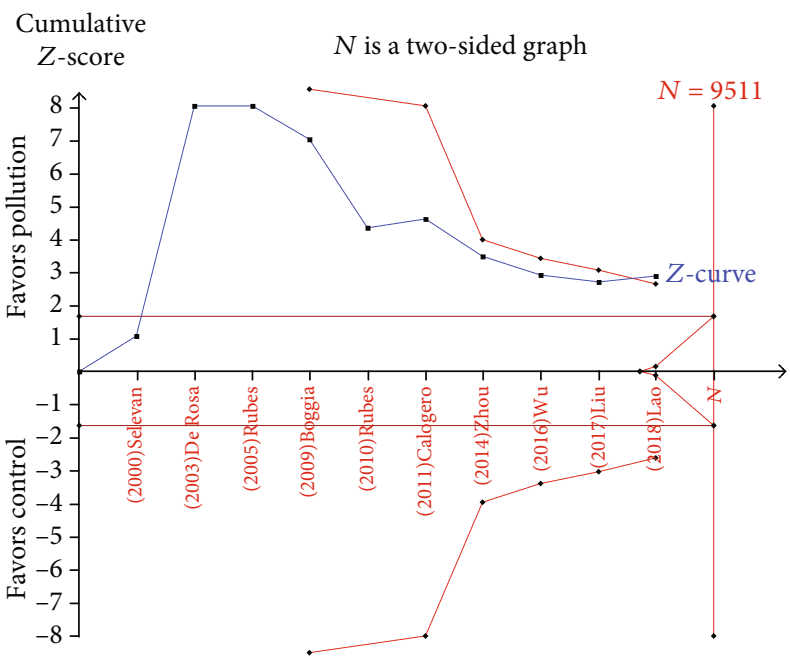

(d)

Figure 4: Trial sequential analysis of the effects of TST. (a-d) TSA of semen volume, sperm concentration, normal morphology rate, and total sperm motility.

causes for the nonsignificant result. First, although most of the enrolled studies (11 studies) focused on the semen concentration, only 5 of them provided total sperm count data. Limited sample size can be one cause for the nonsignificant decrease in total sperm count. Second, the standard deviations of the total sperm count were larger than those of the semen volume and sperm concentration. Based on this, a larger sample size is required to reach statistical significance.

It should be noted that sperm development consists of three different key periods: spermatogenesis, development of sperm motility, and epididymal storage, which correspond to $70-90,10-14$, and $0-9$ days before ejaculation, respectively. Several studies have reported relatively short-term effects (10-14 or 0-9 days before ejaculation) of air pollution on semen parameters but the results were inconclusive. Notably, the exposure assessment of most included studies in the current meta-analysis was based on the information from monitoring stations for at least 90 days before semen sampling, which provided information about relatively long-term effects of air pollution. Further animal researches and epidemiologic studies are required to explore the effects of air pollution on different periods of sperm development.

The current study has several strengths: (1) the sample size was relatively larger, which made our results more reliable; (2) sensitivity analyses, Egger's linear regression tests, and funnel plots indicated that there were no low-quality studies or publication bias; and (3) TSAs were first performed in the current meta-analysis and indicated sufficient evidence that outdoor air pollution can reduce semen volume, sperm concentration, normal morphology rate, and total motility. Notably, compared with those in a previous meta-analysis that included 6 studies [35], the cumulative $Z$-curves in the current meta-analysis crossed the trial sequential monitoring boundaries, meaning the total sample size was more than the estimated information size after adding another 5 studies.

Though this study had a relatively large sample size, several limitations should be stressed: (1) the sources and concentration of the air pollutants varied among the enrolled 
studies, which may increase the heterogeneity between studies and result in potential bias. One reason for this is that pollution levels were different in different regions or seasons, making it difficult to set the same standard. In this meta-analysis, all relevant researches were strictly scanned and most of the studies only provided information about the overall impacts of air pollution. Based on the existing data, the current meta-analysis is aimed at exploring the overall impacts of air pollution on semen quality. (2) The impacts of the single components of the air pollutants were not analyzed because studies provided information concerning single components were limited. Further studies are required to explore the impacts of each component such as $\mathrm{SO}_{2}$ and CO. (3) Other harmful environment urban factors, such as water pollution or electromagnetic waves from cell phones and stations, can decrease semen quality, and their negative influence cannot be objectively separated from the negative influence of toxic air components. (4) Most participants enrolled in this study were Caucasians, and relevant data in Africans and Asians were limited and required further study. (5) Only four studies focused on CASA measures with inconsistent conclusions, and more studies are needed to investigate the effects of air pollution on these indicators. (6) Though the results of TSA indicated a firm association between air pollution and decreased semen volume, concentration, progressive motility, and total motility, more highquality studies are required to offer more individual data.

\section{Conclusion}

Air pollution is associated with decreased semen volume, sperm concentration, motility, and normal morphology rate.

\section{Data Availability}

This is a systematic review and all the data were extracted from the enrolled studies. The original data can be accessed in these studies.

\section{Conflicts of Interest}

The authors declare that they have no conflicts of interest.

\section{Authors' Contributions}

HL conceived and designed the experiments. JZ and ZC searched for and selected the publications. JZ, CM, and JX analyzed the data. JZ, CM, and JX prepared the figures. JZ and ZC contributed materials/analysis tools. JZ and ZC wrote and revised the paper.

\section{Acknowledgments}

This work is supported by the grant from the National Natural Science Foundation of China (Grant No. 81671448).

\section{Supplementary Materials}

Supplement Materials Figure S1: sensitivity of each included study in this meta-analysis. (A-K) Sensitivity analyses of semen volume, sperm concentration, total sperm count, normal morphology rate, progressive motility, total sperm motility, DFI, VCL, VSL, and LIN, respectively. Figure S2: Begg's funnel plots of the publication bias. (A-K) Funnel plots of semen volume, sperm concentration, total sperm count, normal morphology rate, progressive motility, total sperm motility, DFI, VCL, VSL, and LIN, respectively. (Supplementary Materials)

\section{References}

[1] W. Kihal-Talantikite, P. Legendre, P. le Nouveau, and S. Deguen, "Premature adult death and equity impact of a reduction of NO2, PM10, and PM2.5 levels in Paris-a health impact assessment study conducted at the census block level," International Journal of Environmental Research and Public Health, vol. 16, no. 1, p. 38, 2018.

[2] M. A. B. A. Tajudin, M. F. Khan, W. R. W. Mahiyuddin et al., "Risk of concentrations of major air pollutants on the prevalence of cardiovascular and respiratory diseases in urbanized area of Kuala Lumpur, Malaysia," Ecotoxicology and Environmental Safety, vol. 171, pp. 290-300, 2019.

[3] Z. Soleimani, A. Darvishi Boloorani, R. Khalifeh, D. W. Griffin, and A. Mesdaghinia, "Short-term effects of ambient air pollution and cardiovascular events in Shiraz, Iran, 2009 to 2015," Environmental Science and Pollution Research International, vol. 26, no. 7, pp. 6359-6367, 2019.

[4] P. Fu, X. Guo, F. M. H. Cheung, and K. K. L. Yung, "The association between PM2.5 exposure and neurological disorders: a systematic review and meta-analysis," Science of The Total Environment, vol. 655, pp. 1240-1248, 2019.

[5] A. Conforti, M. Mascia, G. Cioffi et al., "Air pollution and female fertility: a systematic review of literature," Reproductive Biology and Endocrinology, vol. 16, no. 1, p. 117, 2018.

[6] R. Lafuente, N. Garcia-Blaquez, B. Jacquemin, and M. A. Checa, "Outdoor air pollution and sperm quality," Fertility and Sterility, vol. 106, no. 4, pp. 880-896, 2016.

[7] J. Jurewicz, E. Dziewirska, M. Radwan, and W. Hanke, “Air pollution from natural and anthropic sources and male fertility," Reproductive Biology and Endocrinology, vol. 16, no. 1, p. 109, 2018.

[8] J. Zhang, B. Yang, Z. Cai, H. Li, T. Han, and Y. Wang, "The negative impact of higher body mass index on sperm quality and erectile function: a cross-sectional study among Chinese males of infertile couples," American Journal of Men's Health, vol. 13, no. 1, 2019.

[9] P. K. Bundhun, G. Janoo, A. Bhurtu et al., “Tobacco smoking and semen quality in infertile males: a systematic review and meta-analysis," BMC Public Health, vol. 19, no. 1, p. 36, 2019.

[10] F. Hardneck, G. Israel, E. Pool, and L. Maree, "Quantitative assessment of heavy metal effects on sperm function using computer-aided sperm analysis and cytotoxicity assays," Andrologia, vol. 50, no. 10, p. e13141, 2018.

[11] E. Ricci, S. Noli, S. Ferrari et al., "Alcohol intake and semen variables: cross-sectional analysis of a prospective cohort study of men referring to an Italian Fertility Clinic," Andrology, vol. 6, no. 5, pp. 690-696, 2018.

[12] A. Agarwal, A. Singh, A. Hamada, and K. Kesari, "Cell phones and male infertility: a review of recent innovations in technology and consequences," International Braz J Urol, vol. 37, no. 4, pp. 432-454, 2011. 
[13] I. Gorpinchenko, O. Nikitin, O. Banyra, and A. Shulyak, "The influence of direct mobile phone radiation on sperm quality," Central European Journal of Urology, vol. 67, no. 1, pp. 6571, 2014.

[14] A. Di Nisio and C. Foresta, "Water and soil pollution as determinant of water and food quality/contamination and its impact on male fertility," Reproductive Biology and Endocrinology, vol. 17, no. 1, p. 4, 2019.

[15] A. Guven, A. Kayikci, K. Cam, P. Arbak, O. Balbay, and M. Cam, "Alterations in semen parameters of toll collectors working at motorways: does diesel exposure induce detrimental effects on semen?," Andrologia, vol. 40, no. 6, pp. 346-351, 2008.

[16] A. E. Calogero, S. la Vignera, R. A. Condorelli et al., "Environmental car exhaust pollution damages human sperm chromatin and DNA," Journal of Endocrinological Investigation, vol. 34, no. 6, pp. e139-e143, 2011.

[17] L. Wu, et al.L. Jin, T. Shi et al., "Association between ambient particulate matter exposure and semen quality in Wuhan, China," Environment International, vol. 98, pp. 219-228, 2017.

[18] Y. Liu, Y. Zhou, J. Ma et al., "Inverse association between ambient sulfur dioxide exposure and semen quality in Wuhan, China," Environmental Science \& Technology, vol. 51, no. 21, pp. 12806-12814, 2017.

[19] S. G. Selevan, L. Borkovec, V. L. Slott et al., "Semen quality and reproductive health of young Czech men exposed to seasonal air pollution," Environmental Health Perspectives, vol. 108, no. 9, pp. 887-894, 2000.

[20] M. De Rosa, S. Zarrilli, L. Paesano et al., "Traffic pollutants affect fertility in men," Hum Reprod, vol. 18, no. 5, pp. 10551061, 2003.

[21] B. Boggia, U. Carbone, E. Farinaro et al., "Effects of working posture and exposure to traffic pollutants on sperm quality," Journal of Endocrinological Investigation, vol. 32, no. 5, pp. 430-434, 2009.

[22] J. Rubes, R. Rybar, P. Prinosilova et al., "Genetic polymorphisms influence the susceptibility of men to sperm DNA damage associated with exposure to air pollution," Mutation Research, vol. 683, no. 1-2, pp. 9-15, 2010.

[23] D. Moher, A. Liberati, J. Tetzlaff, D. G. Altman, and PRISMA Group, "Preferred reporting items for systematic reviews and meta-analyses: the PRISMA statement," Annals of Internal Medicine, vol. 151, no. 4, pp. 264-9, W64, 2009.

[24] J. Zhang, Z. Cai, B. Yang, and H. Li, “Association between outdoor air pollution and semen quality: protocol for an updated systematic review and meta-analysis," Medicine, vol. 98, no. 20, p. e15730, 2019.

[25] J. Rubes, S. G. Selevan, D. P. Evenson et al., "Episodic air pollution is associated with increased DNA fragmentation in human sperm without other changes in semen quality," Human Reproduction, vol. 20, no. 10, pp. 2776-2783, 2005.

[26] N. Zhou, Z. Cui, S. Yang et al., "Air pollution and decreased semen quality: a comparative study of Chongqing urban and rural areas," Environmental Pollution, vol. 187, pp. 145-152, 2014.

[27] X. Q. Lao, Z. Zhang, A. K. H. Lau et al., "Exposure to ambient fine particulate matter and semen quality in Taiwan," Occupational and Environmental Medicine, vol. 75, no. 2, pp. 148154, 2018.
[28] N. Ono, S. Oshio, Y. Niwata et al., "Prenatal exposure to diesel exhaust impairs mouse spermatogenesis," Inhalation Toxicology, vol. 19, no. 3, pp. 275-281, 2007.

[29] H. A. Jeng and L. Yu, "Alteration of sperm quality and hormone levels by polycyclic aromatic hydrocarbons on airborne particulate particles," Journal of Environmental Science and Health. Part A, Toxic/Hazardous Substances \& Environmental Engineering, vol. 43, no. 7, pp. 675-681, 2008.

[30] A. Hammoud, D. T. Carrell, M. Gibson, M. Sanderson, K. Parker-Jones, and C. M. Peterson, "Decreased sperm motility is associated with air pollution in Salt Lake City," Fertility and Sterility, vol. 93, no. 6, pp. 1875-1879, 2010.

[31] J. Zhang, J. Liu, L. Ren et al., "PM2.5 induces male reproductive toxicity via mitochondrial dysfunction, DNA damage and RIPK1 mediated apoptotic signaling pathway," Science of The Total Environment, vol. 634, pp. 1435-1444, 2018.

[32] R. Z. Sokol, P. Kraft, I. M. Fowler, R. Mamet, E. Kim, and K. T. Berhane, "Exposure to environmental ozone alters semen quality," Environmental Health Perspectives, vol. 114, no. 3, pp. 360-365, 2006.

[33] M. Kuchakulla, T. Masterson, H. Arora, S. Kulandavelu, and R. Ramasamy, "Effect of nitroso-redox imbalance on male reproduction," Translational Andrology and Urology, vol. 7, no. 6, pp. 968-977, 2018.

[34] L. Luo, E. Li, S. Zhao et al., "Gasoline exhaust damages spermatogenesis through downregulating $\alpha 6$-integrin and $\beta 1$ integrinin the rat model," Andrologia, vol. 50, no. 7, p. e13045, 2018.

[35] Z. Deng, F. Chen, M. Zhang et al., "Association between air pollution and sperm quality: a systematic review and metaanalysis," Environmental Pollution, vol. 208, pp. 663-669, 2016. 